\title{
USO DE FUNGO ENTOMOPATOGÊNICO PARA CONTROLE DE Aedes aegypti NO CONDOMÍNIO MONDRIAN LIFE, CAMPOS DOS GOYTACAZES - RJ
}

\author{
Adriano Rodrigues de Paula ${ }^{1}$ \\ Anderson Ribeiro ${ }^{2}$ \\ Leila Eid Imad da Silva ${ }^{3}$ \\ Sabrina de Souza Carvalho ${ }^{4}$ \\ Lucas Vardiero Berizonzi ${ }^{5}$ \\ Lorrany Santos da Silva ${ }^{6}$ \\ Richard lan Samuels ${ }^{7}$
}

Resumo: O atual trabalho, com dados preliminares, utilizou, pela primeira vez em Campos dos Goytacazes, Estado do Rio de Janeiro, Brasil, uma armadilha feita de garrafa PET com pano preto impregnado com fungo entomopatogênico Metarhizium anisopliae, denominada "armadilha PET" para o controle populacional de Aedes aegypti. Os testes foram realizados no condomínio "Mondrian Life", em varandas de apartamentos térreos. Os efeitos da utilização da armadilha PET na população de mosquitos de A. aegypti foram avaliados usando ovitrampas. Apartamentos com armadilha PET + fungo tiveram menor número de ovos de A. aegypti em ovitrampas, comparado com tratamento controle. Todos os ovos coletados nas ovitrampas eram de A. aegypti. A diminuição da população do mosquito A. aegypti, provavelmente diminuirá os incômodos causados por esse vetor e a transmissão de dengue, zika e chikungunya.

Palavras-chave: Ovitrampa; Armadilha PET; Fungo entomopatogênico; Teste no campo.

\footnotetext{
${ }^{1}$ Universidade Estadual do Norte Fluminense - Centro de Ciências e Tecnologias Agropecuárias - Laboratório de Entomologia e Fitopatologia, Av. Alberto Lamego, 2000, Campos dos Goytacazes-RJ, Brasil. E-mail: biodepaula@yahoo.com.br.

2 Universidade Estadual do Norte Fluminense - Centro de Ciências e Tecnologias Agropecuárias - Laboratório de Entomologia e Fitopatologia, Av. Alberto Lamego, 2000, Campos dos Goytacazes-RJ, Brasil. E-mail: anderson.ribeirorj@yahoo.com.br.

${ }^{3}$ Universidade Estadual do Norte Fluminense - Centro de Ciências e Tecnologias Agropecuárias - Laboratório de Entomologia e Fitopatologia, Av. Alberto Lamego, 2000, Campos dos Goytacazes-RJ, Brasil. E-mail: imad.saudecoletiva@gmail.com.

4 Universidade Estadual do Norte Fluminense - Centro de Ciências e Tecnologias Agropecuárias - Laboratório de Entomologia e Fitopatologia, Av. Alberto Lamego, 2000, Campos dos Goytacazes-RJ, Brasil. E-mail: sabriinacarvalho8@gmail.com.

5 Universidade Estadual do Norte Fluminense - Centro de Ciências e Tecnologias Agropecuárias - Laboratório de Entomologia e Fitopatologia, Av. Alberto Lamego, 2000, Campos dos Goytacazes-RJ, Brasil. E-mail: lucas.vardiero@hotmail.com.

6 Universidade Estadual do Norte Fluminense - Centro de Ciências e Tecnologias Agropecuárias - Laboratório de Entomologia e Fitopatologia, Av. Alberto Lamego, 2000, Campos dos Goytacazes-RJ, Brasil. E-mail: slorrany633@gmail.com.

7 Universidade Estadual do Norte Fluminense - Centro de Ciências e Tecnologias Agropecuárias - Laboratório de Entomologia e Fitopatologia, Av. Alberto Lamego, 2000, Campos dos Goytacazes-RJ, Brasil. E-mail: richard@uenf.br.
} 